VOL. $4(1971), 1-7$.

\title{
Uniqueness of the Fréchet space topology on certain topological algebras
}

\author{
R. J. Loy
}

It is well known that the complete norm topology on a Banach algebra is not unique in general, though semisimplicity is sufficient (but not necessary) for uniqueness. In this note we consider a class of topological algebras of formal power series which have unique Fréchet space topology. The structure of these algebras in the Banach algebra case will be considered in a later paper.

Let $A$ be an associative algebra over a field $K$, either the real or complex field, having an identity $e$ and carrying a Frechet space topology. A will denote an algebra of formal power series over $A$ in a commutative indeterminate $t$, which we suppose to contain $t$. The elements of $\underline{\underline{A}}$ will be denoted by expressions of the form $\sum a_{i} t^{i}$, where $t^{0}=e, e t=t$. $\underline{\underline{A}}$ will also be supposed to carry a Fréchet space topology, such that the projections $p_{j}: \sum a_{i} t^{i} \leftrightarrow a_{j}$ of $\underline{\underline{A}}$ into $A$ are continuous for each $j \geq 0$.

Note that if $\underline{\underline{A}}$ has an identity and is a unitary left $A$-module under the natural multiplication: $a \sum a_{i} t^{i}=\sum a a_{i} t^{i}$, we may embed $A$ in $\underline{A}$ by the injection $a \rightarrow a+0 . t+\ldots$. Then $A$ can be given the relative topology induced from $\underline{\underline{A}}$, and if the projections are continuous

Received 10 August 1970. This research was partly supported by National Research Council of Canada grant A7655. 
with this topology, $A=\bigcap_{j \geq 1} \operatorname{kerp}_{j}$ is closed, and hence is a Fréchet space under the relative topology. Also, we see that if multiplication in $\underline{\underline{A}}$ is continuous then $A$ is a topological left $A$-module with the relative topology on $A$. It is perhaps of interest to note that this is true more generally. Indeed, in the general case suppose that multiplication in $A$ and $\underline{\underline{A}}$ is continuous and that $\underline{\underline{A}}$ is a left $A$-module under the natural multiplication. Then for $x \in \underline{\underline{A}}$ the map $R_{x}: A \rightarrow \underline{\underline{A}}: a \mapsto$ ax is continuous. For suppose $\left\{y_{n}\right\} \subseteq A, y_{n} \rightarrow 0, R_{x}\left(y_{n}\right) \rightarrow z$. Then $y_{n} x \rightarrow z$ in $\underline{\underline{A}}$ so that $p_{j}\left(y_{n} x\right) \rightarrow p_{j}(z)$ in $A$ for each $j \geq 0$. But $p_{j}\left(y_{n} x\right)=y_{n} p_{j}(x) \rightarrow 0$ for each $j$, whence $p_{j}(z)=0, j \geq 0$, so that $z=0$. The continuity of $R_{x}$ follows by the closed graph theorem. By using the fact that $t$ is not a zero divisor a similar argument shows that for $a \in A$ the map $L_{a}: \underline{\underline{A}} \rightarrow \underline{\underline{A}}: x \mapsto$ ax is continuous. A category argument (cf. [1], Theorem 5) now shows that $\underline{\underline{A}}$ is a topological left A-module.

In [3] I showed, in the case $A^{\prime}$ the complex field and multiplication in $\underline{\underline{A}}$ continuous, that under certain conditions on the projections $\left\{p_{j}\right\}$ derivations on $\underline{\underline{A}}$ are necessarily continuous (the same argument works for more general $A)$, and in [4] that $\underline{\underline{A}}$ has unique complete norm topology if it is a Banach algebra and $A$ is commutative semisimple. By modifying the argument of [3] we now extend this second result. We will say that $\underline{\underline{A}}$ satisfies condition $(E)$ if there is a sequence $\left\{\gamma_{n}\right\}$ of positive reals such that $\left\{\gamma_{n}^{-1} p_{n}\right\}$ is equicontinuous. The first result is the following which, surprisingly, does not require continuity of multiplication in $\underline{\underline{A}}$.

THEOREM 1. Let $B$ be an algebra over $K$ which is a topological algebra under a Fréchet space topology, $\varphi: B \rightarrow \underline{\underline{A}}$ a homomorphism such that $t \in \varphi(B)$, and suppose A satisfies $(E)$. Then $\varphi$ is continuous.

Proof. Since $\left\{p_{j}\right\}$ is a separating family of continuous linear mappings on $\underline{\underline{A}}$ the closed graph theorem shows it is sufficient to prove that $p_{j} \varphi$ is continuous for each $j$. Thus suppose that some $p_{k} \varphi$ is 
discontinuous, and let $k$ be the least integer with this property. If $\left\{\|\cdot\|_{n}\right\}$ is a sequence of seminorms defining the topology on $A$ there is thus some integer $N$ such that $p_{k} \varphi: B \rightarrow\left(A,\|\cdot\|_{N}\right)$ is discontinuous. Let $\left\{\gamma_{n}^{-1} p_{n}\right\}$ be equicontinuous, and take a neighbourhood $V$ of zero in $\underline{\underline{A}}$ such that if $x \in V$ then $\left\|p_{n}(x)\right\|_{N} \leq \gamma_{n}$ for each $n$. Also, since $p_{0} \varphi, \ldots, p_{k-1} \varphi$ are continuous (possibly vacuously so) there is a neighbourhood $U$ of zero in $B$ such that if $x \in U$ then $\left\|p_{i} \varphi(x)\right\|_{N} \leq 1$, $0 \leq i \leq k-1$.

Let $\left\{|\cdot|_{n}\right\}$ be a sequence of seminorms defining the topology on $B$. Since multiplication in $B$ is jointly continuous there is a sequence $\left\{M_{n}\right\}$ of positive integers, and a sequence $\left\{\delta_{n}\right\}$ of positive reals such that if $x, y \in B,|x|_{i}<\alpha,|y|_{i}<\beta, 1 \leq i \leq M_{n}$, where $\alpha \beta<\delta_{n}$, then $|x y|_{j}<1,1 \leq j \leq n$. Finally, take $s \in B$ such that $\varphi(s)=t$, and let $\left\{\mu_{n}\right\}$ be a sequence of positive reals such that $\mu_{n}^{-1} \gamma_{n} \rightarrow 0$. Define inductively a sequence $\left\{x_{n}\right\} \subseteq B$ such that:

(i) $x_{n} \in U$;

(ii) $\left|x_{n}\right|_{i} \leq 2^{-n} \delta_{n} \min _{\substack{1 \leq j \leq n \\ 1 \leq m \leq n}}\left(1+\left|s^{j}\right|_{m}\right)^{-1}$ for $1 \leq i \leq M_{n}$;

(iii) $\left\|p_{k} \varphi\left(x_{n}\right)\right\|_{N} \geq \mu_{n+k}+k+\sum_{i=1}^{n-1}\left\|p_{i+k} \varphi\left(x_{n-i}\right)\right\|_{N}$.

From (ii) it follows that if $m \geq j$ then $\left|s^{m-j} x_{m}\right|_{i}<2^{-m}$ for $1 \leq i \leq m$, and so for each $j$ the series $\sum_{m \geq j} s^{m-j} x_{m}$ converges in $B$. Set $y=\sum_{m \geq 1} s^{m} x_{m}$. Then 


$$
\begin{aligned}
p_{n} \varphi(y) & =p_{n} \varphi\left\{\sum_{m=1}^{n} s^{m} x_{m}+s^{n+1} \cdot \sum_{m \geq n+1} s^{m-n-1} x_{m}\right\} \\
& =p_{n}\left\{\sum_{m=1}^{n} t^{m} \varphi\left(x_{m}\right)+t^{n+1} \cdot \varphi\left(\sum_{m \geq n+1} s^{m-n-1} x_{m}\right)\right\} \\
& =\sum_{m=1}^{n} \sum_{i=0}^{n} p_{n-i}\left(t^{m}\right) p_{i} \cdot\left(x_{m}\right)+0 \\
& =\sum_{i=0}^{n-1} p_{i} \varphi\left(x_{n-i}\right) .
\end{aligned}
$$

Now suppose $n \geq k+2$, so that

$$
\begin{aligned}
\left\|p_{n} \varphi(y)\right\|_{N} & \geq\left\|p_{k} \varphi\left(x_{n-k}\right)\right\|_{N}-\left\{\sum_{i=0}^{k-1}+\sum_{i=k+1}^{n-1}\right\}\left\|p_{i} \varphi\left(x_{n-i}\right)\right\|_{N} \\
& \geq\left\|p_{k} \varphi\left(x_{n-k}\right)\right\|_{N}-k-\sum_{i=1}^{n-k-1}\left\|p_{i+k} \varphi\left(x_{n-k-i}\right)\right\|_{N} \\
& \geq \mu_{n} .
\end{aligned}
$$

Thus taking a non-zero scalar $\lambda$ so small that $\lambda \varphi(y) \in V$ we have $|\lambda| \mu_{n} \leq\left\|p_{n} \varphi(\lambda y)\right\|_{N} \leq \gamma_{n}$, whence $|\lambda| \leq \mu_{n}^{-1} \gamma_{n}$, which is impossible for any sufficiently large $n$ by definition of $\left\{\mu_{n}\right\}$. This gives the desired contradiction.

REMARK. The supposition that $t \in \varphi(B)$ can almost certainly be weakened somewhat, at the cost of complication. However some condition of surjectivity is required, since the result is clearly false in certain cases when $\varphi(B) \subseteq A$.

COROLLARY 1. If $\underline{\underline{A}}$ is itself a topological algebra then every automorphism of $\underline{\underline{A}}$ is continuous.

COROLLARY 2. A has unique Fréchet space topology as a topological algebra.

Proof. Suppose that $\underline{\underline{A}}$ is a topological algebra under a Fréchet space topology determined by seminorms $\left\{|\cdot|_{n}\right\}$ : By Theorem 1 the identity $\operatorname{map}\left(\underline{\underline{A}},\left\{|\cdot|_{n}\right\}\right) \rightarrow\left(\underline{\underline{A}},\left\{\|\cdot\|_{n}\right\}\right)$ is continuous; and hence bicontinuous. 
COROLLARY 3. Any topological algebra A with Fréchet space topology can be isometrically embedded in a topological algebra with unique Fréchet space topology.

Proof. Adjoin an identity $e$ to $A$ if necessary, and if $\left\{\left\|_{n} \cdot\right\|_{n}\right\}$ is a sequence of seminorms defining the topology in $A$ extend them by setting $\|x+\lambda e\|_{n}=\|x\|_{n}+|\lambda|$. Then take $\underline{\underline{A}}$ as the algebra of all formal power series over $A$ such that $\left\|\sum a_{i} t^{i}\right\|_{n}=\sum\left\|a_{i}\right\|_{n}<\infty$ for each $n$, with these seminorms defining the topology. It is easily seen that $\underline{\underline{A}}$ is a topological algebra with this topology, satisfying $(E)$, and so has unique Fréchet space topology as a topological algebra by Corollary 2. The natural injection of $A \rightarrow \underline{\underline{A}}$ is the required embedding.

The fact that $\underline{\underline{A}}$ satisfied (E) played an essential role in the proof of Theorem 1 , and it is of interest to obtain a characterization of this condition. In [4, Lemma 3.1], where the Banach algebra case was considered, the notion of a growth sequence was introduced, and it is precisely this concept which we require here: a sequence $\left\{\sigma_{n}\right\}$ of positive reals is a growth sequence for $\triangleq$ if $\sigma_{n} p_{n}(x) \rightarrow 0$ for each $x \in \underline{\underline{A}}$, convergence in the topology of $A$.

THEOREM 2. Let $A$ be as above, A an algebra of formal power series over A which is a topological algebra under a Fréchet space topology. Then $\triangleq$ satisfies (E) if and only if $\underline{\underline{A}}$ admits a growth sequence.

Proof. Suppose that $\left\{\gamma_{n}^{-1} p_{n}\right\}$ is equicontinuous. Let $U$ be a neighbourhood of zero in $A$, and $V$ a neighbourhood of zero in $\underline{\underline{A}}$ such that $\gamma_{n}^{-1} p_{n}(V) \subseteq U$ for all $n$. Then if $x \in \underline{\underline{A}}$ there is $N$ such that $n^{-1} x \in V$ if $n>N$. Thus if $n>N, n^{-1} \gamma_{n}^{-1} p_{n}(x)=\gamma_{n}^{-} p_{n}\left(n^{1} x\right) \in U$. It follows that $\left\{n^{-1} \gamma_{n}^{-1}\right\}$ is a growth sequence for $\mathrm{A}$.

Conversely, suppose that $\left\{\sigma_{n}\right\}$ is a growth sequence for $\underline{\underline{A}}$. By an argument analogous to [4, Lemma 3.1] and Theorem 1 above it follows 
that the projections are continuous. The Banach-Steinhaus theorem now shows that $\left\{\sigma_{n} p_{n}\right\}$ is equicontinuous.

EXAMPLE. Let $\underline{\underline{A}}$ be the algebra of all formal power series over the complex field, with the coordinate topology. Then $\triangleq$ is a topological algebra under this Fréchet space topology, with continuous projections, but Theorem 2 shows that $\underline{\underline{A}}$ does not satisfy $(E)$. An argument of Scheinberg $[5$, 52] shows that automorphisms on $\underline{\underline{A}}$ are continuous. (Note, however, that $t^{n} \underline{\underline{A}} \neq \underline{\underline{A}}^{n+1}$, so a little care is needed. Indeed, Scheinberg's argument is not applicable to derivations on $\underline{\underline{A}}$; these are continuous by the remark at the end of [2], which is valid in this case.) The same argument shows that $A$ has unique Fréchet space topology under the condition that projections are continuous, though this is easily seen to be true in the general case by use of the closed graph theorem. The present author has been unable to determine whether $\underline{\underline{A}}$ has unique Fréchet space topology as a topological algebra, however the topology as a Q-algebra is unique. For suppose $\underline{\underline{A}}$ is a Q-algebra under a Fréchet space topology $\tau$, and consider the map $\Gamma: x \mapsto x t$. Then $\Gamma$ is a $\tau$-continuous bijection of $\underline{\underline{A}}$ onto $t \underline{\underline{A}}$ and the latter is $\tau$-closed since it is a (in fact the) maximal ideal in $\underline{\underline{A}}$ and $\underline{\underline{A}}$ is a $Q$-algebra under $\tau$. The open mapping theorem shows $\Gamma^{-1}$ is $\tau$-continuous. Since kerp $p_{0}=t_{\underline{A}}$ is $\tau$-closed, $p_{O}$ is $\tau$-continuous, and the relation $p_{j}(x)=p_{j-1}\left(\Gamma^{-1}\left(x-p_{0}(x) e\right)\right), x \in \underline{\underline{A}}, j \geq 1$, gives the $\tau$-continuity of each $p_{j}$ by induction. As remarked above an application of the closed graph theorem now shows that $\tau$ equals the coordinate topology.

\section{References}

[1] Richard Arens, "Linear topological division algebras", BulZ. Amer. Math. Soc. 53 (1947), 623-630.

[2] B.E. Johnson, "Continuity of derivations on commutative algebras", Amer. J. Math. 91 (1969), 1-10.

[3] R.J. Loy, "Continuity of derivations on topological algebras of 
power series", Bull. Austral. Math. Soc. 1 (1969), 419-424.

[4] R.J. Loy, "Uniqueness of the complete norm topology and continuity of derivations on Banach algebras", Tôhoku Math. J. 22 (1970), $371-378$.

[5] S. Scheinberg, "Power series in one variable", J. Math. Anal. App Z. (to appear).

Carleton University,

Ottawa, Canada;

[now at: Department of Pure Mathematics, School of General Studies, Australian National University, Canberra, ACT]. 